\title{
Effect of Social Support on Parenting Stress of Korean Mothers of Children with Cerebral Palsy: An Article Review
}

\author{
Article by Ganiyu Abiodun Adedeji ${ }^{1}$, Caleb Aderemi Adegbenro ${ }^{2}$ \\ ${ }^{I}$ M.Sc. (Physiotherapy), Ph.D. Student, Texila American University, Guyana, South \\ America \\ ${ }^{2}$ Ph.D. (Public Health), Department of Community Health, College of Health Sciences, \\ Obafemi Awolowo University, Ile-Ife, Nigeria \\ E-mail: olabode20032001@yahoo.com ${ }^{1}$
}

\begin{abstract}
Background: Parenting stress in mothers of cerebral palsy children had been linked with common themes such as disturbed social relationships, health problems, financial problems, moments of happiness, worries about future of the child and need for more support services. The objective of this paper was to critically review the article titled: 'Effect of Social Support on parenting Stress of Korean Mothers of Children with Cerebral Palsy' in the Journal: J.Phys.Ther.Sci.

Methods: The review gave a short literature review on the subject. Following this, a summary of the article was given. The article summary summarized the contents of the review. This was followed by a brief analysis of how effective the structure of the article is, investigating how the information is set out and whether or not the reader can have access to it. The presentation of the content of the article including the alignment as well as the format of this article was assessed. The third section was the critique of the article, evaluating its authority and currency. The accuracy, objectivity as well as coverage of the article was as well reviewed. Similarly, the relevance of the content of the article with respect to its title was reviewed. The stability of the article as related to its publication was not left out of the review. This review also carried out an analysis of the figures and tables prior to finally judging the article's accessibility and credibility. Recent advances that are related to the article was briefly explained.

Findings: On the whole, the article was found to be relevant, clear and well written.

Conclusion: The structure, strength, content as well as the limitations of the article were analyzed and critiqued. This article has contributed to the literature considering its valuable critique of current research study on social support and parenting stress.
\end{abstract}

Keywords: Parenting Stress, Social Support, Cerebral palsy, Mothers, Children.

\section{Article source}

Yeon-Gyu J, Yeon-Jae J, Jeong-A B. 2013, 'Effect of Social Support on Parenting Stress of Korean Mothers of Children with Cerebral Palsy', J. Phys. Ther. Sci., 25: 1339-1342.

\section{Introduction}

This paper critically reviews the article titled: 'Effect of Social Support on parenting Stress of Korean Mothers of Children with Cerebral Palsy' in the Journal: J.Phys.Ther.Sci. The review gave a short literature review on the subject. Following this, a summary of the article was given. The article summary summarized the contents of the review. This was followed by a brief analysis of how effective the structure of the article is, investigating how the information is set out and whether or not the reader can have access to it. The presentation of the content of the article including the alignment as well as the format of this article was assessed. The third section was the critique of the article, evaluating its authority and currency. The accuracy, objectivity as well as coverage of the article was as well reviewed. Similarly, the relevance of the content of the article with respect to its title was reviewed. The stability of the article as related to its publication was not left out of the review. This review also carried out an analysis of the figures and tables prior to finally judging the article's accessibility and credibility. Recent advances that are related to the article was briefly explained. On the whole, the article was relevant, clear and well written. An 
appropriate conclusion was drawn at the end of the review and a list of all references cited was given.

\section{Review of literature}

Cerebral palsy has been defined as a permanent, but not irreversible impairment of muscle tone and movement which is caused by a damage of the evolving nervous system before or during birth or in the first months of life [2]. It is otherwise referred to as Little's disease and can as well be defined as a disorder of the central nervous system which constitutes permanent, non-progressive anatomical damage of the motor centers of the brain which manifests in impairments in both mobility and posture [1]. Cerebral palsy has been estimated to be five to ten times more common in under-privileged parts of the world. However, the actual burden is unknown in most low-and middle-income countries as the global incidence has been reported to be between 2 and 3 per 1000 live births [3].

Nimbalkar et al. [4] conducted a study on psychosocial problems among parents of children with cerebral palsy in Western India. The outcome of the study revealed a wide range of psychosocial problems that were experienced by the parents of these children. The problems were associated with common themes such as disturbed social relationships, health problems, financial problems, moments of happiness, worries about future of the child, need for more support services, and lack of adequate number of trained physiotherapists. Another study by Marron et al. [5] found out that the most important predictors of caregiver burden included degree of disability, depression and self-efficacy. The authors concluded that it was necessary to develop interventions to reduce depression and enhance self-efficacy in parents of children with cerebral palsy as one of the primary objectives for minimizing the burden on caregivers of disabled children.

Majnemer et al. [6] conducted a study to describe family distress as reported by parents of children with cerebral palsy as well as to identify factors associated with distress. The outcome of the study showed that family distress measures were modestly associated with motor and cognitive abilities but, more strongly correlated with particular behavioral difficulties. They however found activity limitations across domains to be highly associated with measures of distress. The authors therefore advocated for the need to monitor family functioning at intervals as the child grows and direct necessary resources to optimize child and family well-being. Richard and Alexandra [7] carried out a selective review of interventions designed to reduce stress in parents of children with intellectual disabilities with a focus on group interventions which incorporated various cognitive behavioral techniques. They discovered that research evidence suggested that standard service models such as respite care and case management probably helped to reduce parental stress. They also reported that there were a number of potential practical implications of reducing parental stress for maximizing the efficacy of general parent training interventions and also behavioral programs for children's challenging behaviors.

Olawale et al. [8] conducted a cross-sectional descriptive survey in the Physiotherapy Department of a tertiary hospital in Nigeria. They found that personal problems that were experienced by parents of children with cerebral palsy included loss of job, lack of concentration at work, loss of family joy as well as derangement of financial affairs of the family. The authors however concluded that families caring for children with cerebral palsy generally had a positive attitude towards their children despite the challenges.

\section{Article summary}

The purpose of the article was to investigate the effect of perceived social support on the parenting stress of mothers who have children with cerebral palsy. Background to the topic was given. The methods employed in the study was clearly stated which include consideration of all available published studies and reports on the topic.

The introduction aspect of the article was reviewed where comprehensive definition of cerebral palsy was given with appropriate referencing. The subjects and methods used for the study was as well reviewed in which 181 mothers of children under the age of 12 years, diagnosed with cerebral palsy, who were undergoing rehabilitation therapy at university or rehabilitation hospitals located in Seoul and Gyeonggi-Do province were surveyed. Similarly, the results of the study was also 
reviewed and the discussion of the results was not left out of the review. Finally, a conclusion was drawn from the review.

\section{Article structure}

An abstract to the study summarizing the background of the study, the methods used in the study, the results as well as conclusion was given. The article provided the stance developed by this article including a brief overview of the major points. Included are the rationales for the article and for the research studies it describes. In the article, the paragraphs in the body were not too lengthy; this allows easy access to the information in each of the paragraphs.

Because the article described a research study which was conducted by the authors, it had the conventional information that are usually provided in that kind of study. The sections contained include that related to the background and aims of the study, literature review and subjects/methods for the study. Other sections include the result and discussion. The conclusion can be described as detailed enough and it gave a summary of the main focus of the article. It finally developed future policy and research directions. The references were well cited in-text and were also clearly set out in the literature cited section.

The structure of the article was logically developed looking at it from the total point of view. This was done through the use of relatively short paragraph which has the capability of helping any reader to access the major points without stress. There were many links to the article and this helped in making the information adequately accessible. The availability of link such as that of citations, authors, and references enable any reader to evaluate the worth of the article more effectively. There were also links to headings and subheadings which helped the reader to move through the article faster.

\section{Article critique}

\section{Authority}

The article, published in the journal, J.Phys.Ther.Sci, was found on Google scholar which is a credible research database. The credibility of the authors was established by the fact that they are Physical therapists of high repute working in first class tertiary health institutions in Republic of Korea. The credibility was also ascribed to the fact that the article was open peer reviewed. The article is also an open access one. The availability of many links especially to citations, authors, references, headings and subheadings gives more credibility to the authors.

\section{Accuracy}

The source of the information in this article was a current comprehensive literature review of articles on effect of social support on parenting stress of Korean mothers of children with cerebral palsy. This was supported and backed up by a comprehensive reference list. These sources were cited in-text to support the literature review. The editorial and refereeing processes immensely added to this article's accuracy.

Appropriate references that were cited in discussing the results of the study also added to the articles' accuracy. The declaration section containing acknowledgement, competing interest as well as source of support also make the study to be accurate. The links to other expert sources like journals also contributed to its accuracy.

\section{Currency}

The article was accepted to be published online in the Journal of Physical Therapy Science on May $29^{\text {th }}, 2013$. The article was written and published in a little above four years ago. The literature review it described was relatively current and this article cites many up-to-date references in the body of the text (many of them published in this $21^{\text {st }}$ century). However, there are few references that are of year nineteen hundreds. Therefore, the article is relatively current based on the aforementioned criteria.

\section{Relevance}

This was an academic journal on an academic database that has high credibility in an academic context. It was not written for advertisement or entertainment, but the purpose was to inform 
researchers and students. The article would be relevant to the above-mentioned groups especially those in public health, clinics and health workers in general. It would as well be relevant to both governmental and non-governmental organizations for appropriate policy formulation and implementation on what can be done to alleviate the stress of mothers of children with cerebral palsy. It is very relevant in providing the necessary information that can be useful in the promotion of alleviation of parenting stress both at the local and at the global levels.

\section{Objectivity}

The information contained in the article was objectively developed and well supported with a current research base. All evidences were well referenced and acknowledged as well. The article did not have any trace of bias. This fact was ascertained by the recognition of the fact that the article documents passed through the rigors of research processes including all ethics concerning the research study. The article acknowledged a number of main issues in the paper. The background/literature review gave explanations of the terms that were discussed and their research decision was backed up with appropriate references in the literature. The finding from the study is also relevant to all countries globally. The effectiveness was well discussed and appropriate references were cited.

\section{Stability}

This article is stable as a resource considering the fact that it is published in an academic journal on an academic data base. Its stability is also reinforced by the fact that it is an open access research article that was opened-peer reviewed.

\section{Recent Advances related to 'Effect of Social Support on Parenting Stress of Korean Mothers of Children with Cerebral Palsy'}

Social participation is increasingly of interest in research that investigates the impact of caring for a child with a disability. Dehghan et al. [9] explored social participation among Iranian mothers of children with cerebral palsy. The outcome of the study showed that in the Iranian context, mothers of children with cerebral palsy are facing many challenges to social participation and seem to have been neglected by the health-care system.

In a very recent study, Yongli et al. [10] investigated the mediating effect of social support on the relationship between parenting stress and life satisfaction in Chinese mothers of children with cerebral palsy $(N=369)$. The results revealed that family support and friend support, but not significant-other support, had mediating effects on the relationship between parenting stress and life satisfaction. However, they found out that the mediating effect of friend support was equal to that of family support. It was suggested that the focus should be on reducing parenting stress and increasing support from family and friends to help improve life satisfaction in mothers of children with cerebral palsy.

A study by Heykyung and Eun-Kyoung [11] examined caregiver burden and social supports perceived by mothers raising children with developmental disabilities in South Korea. Mothers residing in the metropolitan areas of Seoul responded to the mail survey. Results showed a high level of overall burden, particularly in financial domains. Greater subjective caregiver burden for these mothers was associated with: increased disability-related costs; maternal factors like being younger and having higher educational attainment; and less social support. Parvinian and his coworkers [12] found out that applying a designed- supportive program was capable of influencing positively the mental health status of the mothers who had children with cerebral palsy. Because of their findings, they suggested that designed-supportive programs should be applied as an effective method to promote mental health of mothers of children with cerebral palsy.

Grace and Rumaya [13] conducted a study to determine the relationships between maternal appraisal, social support and parenting stress among mothers of children with cerebral palsy in Selangor, Malaysia. The data were collected through self-administered questionnaire with the participation of a total of forty-two mothers of children with cerebral palsy. The findings of this study showed significant relationships between maternal appraisal and parenting stress, whereby negative appraisal and parenting stress were positively correlated. However, mothers' positive appraisal correlated negatively with parenting stress. Similar negative correlation was also identified between perceived social support and parenting stress. The outcome of the study revealed that maternal 
appraisal plays a significant role in determining parenting stress among mothers of children with cerebral palsy. Specifically, negative appraisal was found to be the strongest predictor of maternal parenting stress. The authors finally reiterated the importance of boosting positive appraisal and preventing negative appraisal as measures in reducing the maternal parenting stress among such mothers.

A study to identify the level of burden and social support of mothers with cerebral palsy children, and to determine whether providing social support is an effective strategy to relieve the burden of those mothers was carried out by Eun-Sook and Hee-Soon [14]. The outcome showed that the burden of mothers was related to the Gross Motor Function Classification System (GMFCS) of the cerebral palsy children. Social support was also negatively correlated and GMFCS was positively correlated with the burden of mothers. The study demonstrated that social support which is an important factor relating to burden imposed on mothers of cerebral palsy children, can be considered as an intervening variable which can contribute to decrease of burden in mothers of such children.

Jermaine et al. [15] conducted a research to establish the well-being of caregivers of children with Cerebral Palsy living in high-density areas of Harare, Zimbabwe. The aim of the authors also included identifying the factors that might be predictive of caregivers' well-being. The result of the study showed that caregivers reported considerable caregiver burden. Many of them experienced some form of pain and depression and they indicated that they were overwhelmed by the caregiving role. The authors however suggested that caregivers must be monitored routinely for their level of distress with an urgent need to provide them with support.

\section{Conclusion}

This article review has summarized and critically reviewed the article titled 'Effect of Social Support on Parenting Stress of Korean Mothers of Children with Cerebral Palsy which was authored by Yeon-Gyu J, Yeon-Jae J and Jeong-A B. The structure, strength, content as well as the limitations of the article were analyzed and critiqued. This article has contributed to the literature considering its valuable critique of current research study on social support and parenting stress.

\section{References}

[1]. Dehghan L, Dalvandi A, Rassafiani M, Hosseini SA, Dalvand H, Baptiste S (2015). Social participation experiences of mothers of children with cerebral palsy in an Iranian context. Aust Occup Ther J. 62(6):410-9. doi: $10.1111 / 1440-1630.12215$.

[2]. Eun-Sook K and Hee-Soon K (2009). Burden and Social Support of Mothers with Cerebral Palsy Children. The Korean Journal of Rehabilitation Nursing. 12.

[3]. Grace A H and Rumaya J (2017). Maternal Appraisal, Social Support and Parenting Stress among Mothers of Children with Cerebral Palsy. International Journal for Studies on Children, Women, Elderly and Disabled. 1.

[4]. Hodapp MR (1998). Advancing Finding, Theories, and Methods Concerning Children with Disabilities. Athens: Cambridge University Press.

[5]. Heykyung O. and Eun-Kyoung OL. (2009). Caregiver Burden and Social Support among Mothers Raising Children with Developmental Disabilities in South Korea. International Journal of Disability, Development and Education. 56(2): 149-167.

[6]. Jermaine MD, Jennifer J and Tecla M (2015). Caring for a child with Cerebral Palsy: The experience of Zimbabwean mothers. African Journal of Disability. 4(1). http://dx.doi.org/10.4102/ajod.v4i1.168.

[7]. Marrón, EM, Redolar-Ripoll D., Boixadós M., Nieto, R., Guillamón N., Hernández E. (2013). Burden on caregivers of children with cerebral palsy: Predictors and related factors. Universitas Psychologica, 12(3), 767-777.

[8]. Majnemer A, Shevell M, Law M, Poulin C, Rosenbaum P. (2012). Indicators of distress in families of children with cerebral palsy. Disabil Rehabil, 34(14), 1202-1207.

[9]. Nimbalkar S, Raithatha S., Shah R., Panchal DA. (2014). A Qualitative Study of Psychosocial Problems among Parents of Children with Cerebral Palsy Attending Two Tertiary Care Hospitals in Western India. ISRN Family Med, doi: 10.1155/2014/769619.

[10]. Olawale OA, Abraham ND. and Raphael K.K. (2013). Psychological impact of cerebral palsy on families: The African perspective. J Neurosci Rural Pract, 4(2), 159-163.Ozmen M, Caliskan M, Apak S., Gokcay G. (1993). 8-Year clinical experience in cerebral palsy. J Trop Pediatr, 39, 52-54. 
DOI: $10.21522 /$ TIJPH.2013.05.04.Art066

ISSN: $2520-3134$

[11]. Parvinian AM, Kermanshahi SM, Sajedi F (2012). The Effect of a supportive program on mental health of mothers of children with cerebral palsy. http://osub.mums.ac.ir/osub/nilfr/nmjournal/index.php.

[12]. Richard PH. and Alexandra B. (2004). Practitioner review: Stress intervention for parents of children with intellectual disabilities. Journal of Child Psychology and Psychiatry, 45(8), 1338-1349.

[13]. Thanou E, Tsiou C, Kattami, C, Chrousos, GP, \& Darviri, C (2016). A Stress Management and Health Promotion Intervention for Parents of Children with Cerebral Palsy and Motor Disability at the Infant Department of the Greek Center for the Protection and Rehabilitation of the Disabled (ELEPAP). QuasiExperimental Study. Psychology, 7, 557-564.

[14]. Yongli W, Zhaoming H and Feng K. (2017). Parenting stress and life satisfaction in mothers of children with cerebral palsy: The mediating effect of social support. Journal of Health Psychology. 